\title{
Reduced gray matter volume in male adolescent violent offenders
}

\author{
Ying-Dong Zhang ${ }^{1,2}$, Jian-Song Zhou ${ }^{1,2}$, Feng-Mei Lu ${ }^{3,4}$, Xiaoping Wang ${ }^{\text {Corresp. 1, } 2}$ \\ ${ }^{1}$ Department of Psychiatry \& Mental Health Institute of the Second Xiangya Hospital, Central South University, Changsha, Hunan, China \\ 2 National Clinical Research Center on Mental Disorders \& National Technology Institute on Mental Disorders, Hunan Key Laboratory of Psychiatry and \\ Mental Health, Changsha, Hunan, China \\ ${ }^{3}$ The Clinical Hospital of Chengdu Brain Science Institute, MOE Key Lab for Neuroinformation, University of Electronic Science and Technology of China, \\ Chengdu, Sichuan, China \\ 4 School of Life Science and Technology, Center for Information in Medicine, University of Electronic Science and Technology of China, Chengdu, Cichuan, \\ China \\ Corresponding Author: Xiaoping Wang \\ Email address: xiaop6@csu.edu.cn
}

Background: Previous studies reported that reduced gray matter volume (GMV) was associated with violent-related behaviors. However, the previous studies were conducted on adults and no study has studied the association between GMV and violent behaviors on adolescents. The purpose of the study was to investigate GMV's effects in adolescent violent offenders based on a Chinese Han population, which can address the problem of possible confounding factors in adult studies. Methods: We recruited 30 male adolescent violent offenders and 29 age- and sex-matched healthy controls (HCs). Differences in both whole-brain and GMV were evaluated using voxel-based morphometry (VBM). We assessed the accuracy of VBM using the receiver operating characteristic curve (ROC) and discriminant analysis. Results: Compared with HCs, the male adolescent offenders showed significantly reduced GMV in five cortical and subcortical brain regions, including the olfactory cortex, amygdala, middle temporal gyrus and inferior parietal lobe in the left hemisphere, as well as the right superior temporal gyrus. Both ROC curve and discriminate analyses showed that these regions had relatively high sensitivities (58.6\%-89.7\%) and specificities (58.1\%-74.2\%) with 76.7\% classification accuracy. Conclusions: Our results indicated that reduced volume in the frontal-temporal-parietal-subcortical circuit may be closely related to violent behaviors in male adolescents, which might be an important biomarker for detecting violent behaviors in male adolescents. 


\section{Reduced gray matter volume in male adolescent}

2 violent offenders

3

4 Ying-Dong Zhang ${ }^{1,2}$, Jian-Song Zhou ${ }^{1,2}$, Feng-Mei Lu ${ }^{3}$, Xiao-Ping Wang ${ }^{1,2^{*}}$

$5 \quad{ }^{1}$ Department of Psychiatry \& Mental Health Institute of the Second Xiangya Hospital, Central

6 South University, Changsha, Hunan province, China.

$7 \quad 2$ National Clinical Research Center on Mental Disorders \& National Technology Institute on

8 Mental Disorders. Hunan Key Laboratory of Psychiatry and Mental Health, Changsha, Hunan

9 province, China.

$10{ }^{3}$ The Clinical Hospital of Chengdu Brain Science Institute, MOE Key Lab for Neuroinformation, 11 University of Electronic Science and Technology of China, Chengdu, China.

$12{ }^{4}$ School of Life Science and Technology, Center for Information in Medicine, University of

\section{Corresponding Author:}

Xiao-Ping Wang ${ }^{1,2}$

Department of Psychiatry \& Mental Health Institute of the Second Xiangya Hospital, Central South University; National Clinical Research Center on Mental Disorders \& National Technology Institute on Mental Disorders, Hunan Key Laboratory of Psychiatry and Mental Health, Changsha, Hunan 410011, China (e-mail: xiaop6@126.com, xiaop6@csu.edu.cn)

\section{Abstract}

Background: Previous studies reported that reduced gray matter volume (GMV) was associated with violent-related behaviors. However, the previous studies were conducted on adults and no study has studied the association between GMV and violent behaviors on adolescents. The purpose of the study was to investigate GMV's effects in adolescent violent offenders based on a Chinese Han population, which can address the problem of possible confounding factors in adult studies.

Methods: We recruited 30 male adolescent violent offenders and 29 age- and sex-matched healthy controls (HCs). Differences in both whole-brain and GMV were evaluated using voxelbased morphometry (VBM). We assessed the accuracy of VBM using the receiver operating characteristic curve (ROC) and discriminant analysis.

Results: Compared with HCs, the male adolescent offenders showed significantly reduced GMV in five cortical and subcortical brain regions, including the olfactory cortex, amygdala, middle temporal gyrus and inferior parietal lobe in the left hemisphere, as well as the right 
36

37

38

39

40

41

42

43

44

45

46

47

48

49

50

51

52

53

54

55

56

57

58

59

60

61

62

63

64

65

66

superior temporal gyrus. Both ROC curve and discriminate analyses showed that these regions had relatively high sensitivities $(58.6 \%-89.7 \%)$ and specificities $(58.1 \%-74.2 \%)$ with $76.7 \%$ classification accuracy.

Conclusions: Our results indicated that reduced volume in the frontal-temporal-parietalsubcortical circuit may be closely related to violent behaviors in male adolescents, which might be an important biomarker for detecting violent behaviors in male adolescents.

Keywords: Violence, brain structural abnormalities, VBM, ROC, discriminate analyses

\section{Introduction}

Add your introduction here. Violence is a global public health problem that causes great personal sufferings as well as social problems (Krug et al., 2002). The World Health Organization report shows that the global homicide rate in males is significantly higher than in females (13.6 versus 4.0 per 100,000 population) in all age groups (Krug et al., 2002). Among different age groups, adolescents and young adults (15-29 years old) have the highest homicide rates: 19.4 for males and 4.4 for female per 100,000 population (Krug et al., 2002). These reports suggest that the violence problem is more severe in adolescents and young adults, especially males. Moreover, the youth violence has an extremely negative impact on communities and social stability (Greenwood, 2008). Therefore, early identification and management of violent behavior among adolescents not only can save their lives, but also can prevent the development of criminal behaviors in adulthood, thus reducing the burden of crime on society (Greenwood, 2008).

The relationship between abnormal brain structures and violent behaviors has long been noted. It was reported that damage to certain brain areas was related to aggression and violence (Grafman et al., 1996). A longitudinal brain study also revealed that abnormal brain structure was associated with an increased amount of criminal behaviors (McKinlay et al, 2014). In addition, it was reported that the abnormal brain structure and function were key biological risk factors for violence (Rosell and Siever, 2015). Previous identification of persons who were prone to violent behavior was primarily based on personality assessments and electroencephalography. In recent years, the development of noninvasive imaging tools, such as magnetic resonance imaging (MRI), single photon emission computed tomography, and positron emission tomography, has 
67 offered new ways for identifying individuals who are at risk of engaging in violent behavior

68 (Leutgeb et al, 2016; Puri et al, 2018).

69

70 Evidence from limited structural neuroimaging studies has indicated that reduction of the gray

71 matter volume (GMV) in several brain regions that subserve both emotion processing and

72 behavior regulation, such as the orbitofrontal cortex (OFC), prefrontal cortex, medial temporal

73 cortex, amygdala, basal ganglia, anterior cingulate cortex, was closely related to violent-related

74 behaviors (Leutgeb et al., 2016; Rosell and Siever, 2015). The brain develops steadily in terms of

75 both structure and function throughout childhood and early adulthood. The developmental

76 trajectory of GMV is complicated and heterogeneous between different brain regions. For

77 example, the GMV in cortex and some subcortical structures (such as the caudate) follows an

78 inverted U-shape developmental course (Giedd and Denker, 2015). In comparison, this

79 developmental trajectory has not been found in other subcortical structures (such as amygdala

80 and hippocampus) (Giedd and Denker, 2015). Moreover, most of the previous studies regarding

81 violence-related structural abnormality were based on adults (Leutgeb et al., 2016; Rosell and

82 Siever, 2015). Only a few studies in children and adolescents have indicated that decreased

83 GMV of several brain regions may be a constant marker for violence-related behaviors (Sterzer

84 and Stadler, 2009; Vloet et al., 2008). For example, lower amygdala volume was reported to be

85 associated with increased violent behaviors from childhood to adulthood and even predicted

86 future violence (Pardini et al., 2014). More importantly, an adolescents based study can avoid

87 possible confounding factors that are commonly seen in adult based studies, such as the shorter

88 duration of illness, medication naïve and lower rates of comorbidities. Thus, studying structural

89 neuroimaging in adolescents has advantages over that in adults.

91 Moreover, most previous studies on violent behaviors usually were conducted in patients with

92 psychiatric disorders such as conduct disorder (CD), antisocial personality disorder (APD),

93 borderline personality disorder (BPD), attention-deficit hyperactivity disorder (ADHD) or

94 schizophrenia, with aggression, impulsivity, psychopathy or callous-unemotional (CU) traits

95 (Laakso et al, 2002; Schiffer et al, 2011). Only a few previous studies investigated the

96 neuroimaging characteristics of impulsive or aggressive behaviors in healthy subjects (Boes et

97 al., 2009; Boes et al., 2008; Ducharme et al., 2011; Matsuo et al., 2009a; Matthies et al., 2012; 
98 Pardini et al., 2014). $\{\{\{$ Comments: Can you remove some of the citations on healthy-subject

99 based studies? You are saying most studies are based on mental disorder patients but according 100 to your citations, there are more healthy-subject based studies. \} \} F Furthermore, most previous 101 violence-related brain studies were based on males (Boes et al., 2009; Boes et al., 2008; De Brito 102 et al., 2009; Ducharme et al., 2011; Ermer et al., 2013; Fairchild et al., 2011; Huebner et al., 103 2008; Sterzer et al., 2007).

104

105 The aforementioned violence-brain studies are limited either by using adults or by lacking 106 emphasis on healthy subjects. To fill the gap of the aforementioned GMV-violence studies, we 107 investigated the effects of abnormal brain cerebral structures on violent behaviors and focused 108 the study on healthy male adolescents. More specifically, using voxel-based morphometry 109 (VBM) and region of interest (ROI)-based methods, we analyzed the differences in GMV and 110 white matter volume (WMV) between violent offenders and controls.

111

\section{Materials \& Methods}

\section{Participants}

114 A total of 30 male adolescent offenders (aged from 15 to 18 years), who had been convicted of 115 aggressive behavior in court (4 murder or manslaughter, 15 intentional injury, and 11 robbery 116 with injury), and 29 age- and gender-matched healthy controls (HCs) were recruited. Male 117 adolescent offenders were recruited from the Youth Detention Centre (YDC), Hunan province, 118 China. All of them had been convicted of either homicide or assault. Male HCs were students, 119 recruited from a middle school in Changsha and further screened by research psychiatrists to 120 exclude subjects with past mental disorders. All participants were right-handed, and had no 121 history of neurological impairments. The substance abusers were excluded based on their urine 122 analysis, self-report and family informant report within the last 3 months.

124 All interviews were conducted by research psychiatrists. The Chinese version of the Schedule for 125 Affective Disorders and Schizophrenia for School-Age Children Present and Lifetime version 126 (K-SADS-PL) (Shanee et al., 1997) was used for detecting current and past psychiatric problems 127 according to Diagnostic and Statistical Manual of Mental Disorders (DSM)-IV criteria. We excluded 2 adolescent offenders who were diagnosed with either current or past psychiatric 
129 disorders. In addition, we also collected the information of criminal history, psychosocial history, 130 alcohol or other drug use, family history, history of psychiatric, and other medical treatments for 131 each participant.

132

133 Signed written informed consent was obtained from all participants and all study procedures 134 were approved by Institutional Review Board of the second Xiangya Hospital, Central South 135 University.

\section{MRI data acquisition}

138 The structural three-dimensional (3D) T1-weighted MRI images were acquired using a 3.0 Tesla 139 Siemens Vision scanner at the Magnetic Resonance Center of the Hunan Provincial People's 140 Hospital. A 3D magnetization-prepared rapid-acquisition gradient echo (3D MPRAGE) sequence was used with the following parameter settings: Repetition Time (TR) $=2000 \mathrm{~ms}$; Echo Time $(\mathrm{TE})=3.36 \mathrm{~ms}$; flip angle $=9^{\circ}$; pixel matrix $=256 \times 256$, Field of view $(\mathrm{FOV})=256 \times 256$ $\mathrm{mm} 2$, voxel size $=1 \times 1 \times 1 \mathrm{~mm} 3$, and number of slices $=144$.

\section{Data processing and statistical analysis}

Demographic and clinical characteristics analysis: Two-sample t-tests, chi-square test, and the

Fisher exact test were used to analyze the sociodemographic and clinical characteristics of the participants. A p-value less than 0.05 was considered as statistically significant. All analyses were performed using the Statistical Package for Social Sciences (SPSS) version 16.0 (SPSS, Chicago, Ill., USA).

$\boldsymbol{V B M}$ : For the morphometric analysis, the VBM8 (http://dbm.neuro.uni-jena.de/vbm8/), a toolbox of SPM8 (http://www.fil.ion.ucl.ac.uk/spm/soft-ware/spm8/) software, was applied with the DARTEL algorithm. For each participant, the origin of the images was first manually aligned to the anterior commissure for a better registration. The structural images were then segmented into gray matter (WM), white matter (WM) and cerebrospinal fluid (CSF) using a standard unified segmentation model in SPM8. Fig 1 shows the segmentation results of one subject. After that, the DARTEL approach was performed for registration, normalization and modulation in the 
160 fields from the segmentation procedure, and all the individual deformation filed map was 161 registered to the new template. The GM images were then normalized to a study-specific 162 template in Montreal Neurological Institute (MNI) space. To preserve the volume of GM, 163 Jacobian determinants are used to modulate the voxel values of GM. After that, the images were 164 smoothed with the Gaussian kernel of $8 * 8 \mathrm{~mm}$. After spatial preprocessing, the normalized, 165 modulated and smoothed GM and WM images were used for further statistical analyses.

166

167 Put Figure 1 above here.

168

169

In our study, both whole-brain level and region-of-interest (ROI) level VBM analyses were performed. The whole-brain level analysis is automated and unbiased, making no assumptions of about any regions of particular interest. However, this technique requires a great number of subjects to achieve a statistical significance and therefore changes in smaller structures may be difficult to identify due to the small sample size. To address this problem, a secondary ROI level analysis is often used to test the differences only in the voxels that are deemed of interest by an a priori hypothesis. The ROI level analysis can be used to corroborate the findings of previous whole-brain level studies, or those obtained during the whole-brain analysis. This is of special importance in studies with a small sample size(Husain et al., 2011).

178

Whole brain analysis: Two sample T-tests were performed to evaluate the significance of the data using the initial threshold of $\mathrm{P}=0.001$. The $\mathrm{p}$-values were then adjusted using false discovery rate $($ FDR) correction (with cluster size $>100)$ to correct multiple testing. A FDRcorrected $p$-value $<0.05$ was considered as statistically significant.

ROI analysis: Brain regions that showed significant differences in GMV between two groups in the whole brain analysis were identified as significant ROIs. The main advantage of using a priori ROI test over a whole brain analysis is that it reduces type I errors by narrowing down the statistical tests to only a few ROIs(Husain et al., 2011). For each participant, the mean GMV of each ROI was then extracted using an approach described previously(Santillo et al., 2013). We then used the receiver operating characteristic curve (ROC) analyses to investigate the sensitivity and specificity of the detected abnormalities in GMV, which enabled us to evaluate the 
191 discriminative power of these abnormalities in identifying the violent adolescent offenders. In 192 addition, as suggested in one previous study (Jednorog et al., 2014), a further Fisher discriminant 193 analysis was performed to predict whether a participant was a violent offender according to the 194 individual's GMV for those ROIs which exhibited significant differences $(p<0.001)$ in GMV 195 between the two groups.

196

197

\section{Results}

198

Demographic and clinical characteristics

199

The results of Two-sample T-tests were presented in Table 1. As shown in Table 1, there is no 200 significant difference between the adolescent offenders and HCs in terms of age, total

201

202

203

204

205

206

207

208

209

210

211

212

213

214

215

216

217 Put Table 2 above here.

218 Put Figure 2 above here.

219

220

\section{Put Table 1 above here.}

\section{Whole brain analysis}

\section{6}

\section{ROI Analysis} intracranial volume, GMV, and WMV (all p-values $>0.05$ ). In comparison, the educational level of the adolescent offenders was significantly lower than that of the HCs $(7.5 \pm 2.4$ vs $10.0 \pm 0.0$ years; $\mathrm{t}=-5.10 ; \mathrm{p}$-value $<0.01$, which was adjusted in the following analyses. The educational difference is due to that most of the adolescent offenders had dropped out from the junior high school, while all HCs were students from senior schools.

The result of whole-brain analysis was presented in Table 2. Compared to the HCs, the adolescent offenders showed significantly lower GMV ( $<<0.05$, FDR corrected) in the olfactory cortex, amygdala, middle temporal gyrus and inferior parietal lobe in the left hemisphere, as well as the right superior temporal gyrus (Figure 2). In comparison, there was no significant difference in GMV of the whole brain between the offenders and HCs. Similarly, no significant difference in WMV was observed between the two groups. 
221 Five ROIs were identified as significant based on the whole-brain analysis, including the left 222 olfactory cortex, left amygdala, left middle temporal gyrus, left inferior parietal lobe, and the 223 right superior temporal gyrus. A further ROC analysis was conducted on these ROIs and the 224 results were presented in Table 3. The sensitivity, specificity and Area Under the Curve (AUC) 225 values were $58.6 \%, 67.7 \%$ and 0.642 for the left olfactory cortex; $86.2 \%, 74.2 \%$ and 0.834 for 226 the left amygdala; $79.3 \%, 58.1 \%$ and 0.721 for the left middle temporal gyrus; $86.2 \%, 74.2 \%$ and 2270.834 for the left inferior parietal lobe; and $89.7 \%, 64.5 \%$ and 0.790 for the right superior 228 temporal gyrus. It was suggested that the AUC values can be interpreted as excellent if AUC $229 \geq 0.90$; good if $0.90>\mathrm{AUC} \geq 0.80$; fair if $0.80>\mathrm{AUC} \geq 0.70$; poor if $0.70>\mathrm{AUC} \geq 0.60$; and no 230 effect if AUC $<0.60$ (Linden, 2006). Hence, our results suggested that except for the left olfactory 231 cortex, GMV reduction of each of the other four ROIs represented a fair or good biomarker for 232 violence in juveniles.

233

\section{Put table 3 above here.}

235

236 These five ROIs were then used for a discriminant analysis. The overall classification accuracy 237 was 76.7\%: 75.9\% accuracy for the HC group versus 77.4\% accuracy for violent adolescent 238 offenders. The validity of using GMV to distinguish two groups was supported by Wilk's 239 lambda $=0.57(\mathrm{df}=4, \mathrm{p}<0.001)$.

240

\section{Discussion}

242 To our best knowledge, this study represented the first attempt to use the VBM method for 243 identifying brain structural abnormalities in a cohort of male violent adolescents with no history 244 of mental disorders. We found that male adolescent offenders had a reduced GMV in the 245 olfactory cortex, amygdala, middle temporal gyrus and inferior parietal lobe in the left 246 hemisphere, and in the right superior temporal gyrus than male HCS. These findings provide 247 evidence that male violent adolescent offenders exhibit reductions of GMV in several brain 248 regions.

249

250 The pathophysiology of violence-related behaviors has not been understood clearly. Several lines 251 of studies showed that violent behaviors were related with structural or functional abnormalities 
252 in several brain regions that subserve both emotion processing and behavior regulation (Leutgeb 253 et al., 2016; Rosell and Siever, 2015). Amygdala is a subcortical structure of limbic system that 254 plays an essential role in the integration of a wide range of sensory and motivationally salient 255 stimuli, as well as in transmission of this information to various cortical and subcortical regions. 256 These processes underlie the amygdala's essential role in fear mediating, defensive reactions, 257 emotional learning, and motivation. Amygdala has long been considered as the most important

258

259

260

261

262 263

264

265

266

267

268

269

270

271

272

273

274

275

276

277

278

279

280

281

282 neural region for violence-related behaviors (Lane et al., 2011; Perathoner et al., 2016). Similar to the finding in our current study, several previous studies found a decreased amygdala volume in juvenile subjects prone to violence than their healthy peers(Fairchild et al., 2011; Huebner et al., 2008; Sterzer et al., 2007; Stevens and Haney-Caron, 2012). One longitudinal study reported that a lower amygdala volume was a significant factor for detecting increased violent behaviors from childhood to adulthood (Pardini et al, 2014). Further, the frontal cortex, especially the OFC, exhibits the most robust reciprocal anatomical connections with the amygdala, which receives limbic inputs from amygdala and other medial temporal areas as well as sensory inputs (Leutgeb et al, 2016). Thus, frontal cortex may integrate sensory information with affective signals, inhibitory control signals from other areas and is considered as the trigger point of violencerelated behaviors (Lane et al., 2011; Rosell and Siever, 2015). Several previous studies reported decreased GMV of OFC in subjects prone to violence (Bertsch et al., 2013; Boes et al., 2009; de Oliveira-Souza et al., 2008; Huebner et al., 2008; Laakso et al., 2002; Tiihonen et al., 2008), negative correlation between GMV of OFC and impulsive (Kumari et al., 2009; Matsuo et al., 2009a), aggressive (Gansler et al., 2009) or psychopathic (Ermer et al., 2012, 2013) level. These results support our finding of decreased GMV of olfactory cortex, as part of the OFC, in violent adolescents. In addition, the temporal cortex and the parietal cortex, both of which are closely connected to the limbic system, are important for regulating the affective nature of interpersonal experiences and play a pivotal role in the development of emotional behavior (Soderstrom et al., 2002). Previous studies showed decreased GMV of several regions of the temporal or the parietal lobe, such as superior temporal cortex (Bertsch et al., 2013; de Oliveira-Souza et al., 2008; Muller et al., 2008; Stevens and Haney-Caron, 2012), middle temporal cortex (Bertsch et al., 2013), inferior temporal cortex (Bertsch et al., 2013; Gregory et al., 2012; Stevens and HaneyCaron, 2012), hippocampus (Barkataki et al., 2006; Huebner et al., 2008; Stevens and HaneyCaron, 2012; Yang et al., 2010), parahippocampal gyrus (Bertsch et al., 2013; Stevens and 
283 Haney-Caron, 2012; Yang et al., 2010), temporal pole (Bertsch et al., 2013), inferior parietal 284 cortex(Tiihonen et al., 2008), postcentral cortex (Bertsch et al., 2013; Stevens and Haney-Caron, 285 2012; Tiihonen et al., 2008), angular gyrus (Puri et al., 2008) and supramarginal gyrus (Puri et 286 al., 2008; Stevens and Haney-Caron, 2012) in subjects with a tendency toward violence. Also, 287 we found low GMV of three regions in the temporal or parietal lobe, the middle temporal gyrus, 288 the superior temporal gyrus and the inferior parietal lobe in adolescent violent offenders, which 289 were consistent with these previous studies. tOur results and previous literatures indicate that 290 reduced GMV in the frontal-temporal-parietal-subcortical circuit may lead to difficulties in 291 suppressing expressions of emotion, which may further lead to inappropriate, or even violent 292 behaviors.

293

294 Low GMV of several areas in the frontal-temporal-parietal-subcortical circuit was found to be a 295 marker for violence-related behaviors both in juveniles (Boes et al., 2009; Boes et al., 2008; Bussing et al., 2002; Ducharme et al., 2011; Ermer et al., 2013; Fairchild et al., 2011; Huebner et al., 2008; Kruesi et al., 2004; Sterzer et al., 2007) and in adults(Barkataki et al., 2006; Bertsch et al., 2013; de Oliveira-Souza et al., 2008; Ermer et al., 2012; Gansler et al., 2009; Gregory et al., 2012; Kumari et al., 2009; Laakso et al., 2002; Matsuo et al., 2009a; Matsuo et al., 2009b;

300

301 302

303 304 305 306 307 308 309 310 311 312 Matthies et al., 2012; Muller et al., 2008; Pardini et al., 2014; Puri et al., 2008; Raine et al., 2000; Tiihonen et al., 2008; Yang et al., 2010; Yang et al., 2005; Zhang et al., 2013).

We evaluated the discriminative power of both GMV and the identified brain regions in classifying violent offenders and controls. The ROC analysis with VBM on each region revealed that reduced GMV in four regions (left amygdala, left middle temporal lobe, left inferior parietal lobe, and right superior temporal gyrus) represented either a fair or a good biomarker of violence in juveniles. Further discriminant analysis indicated that reduced GMVs of the five regions could predict whether a subject was a violent offender with $76.7 \%$ accuracy, suggesting that reduced GMV of the five regions could be regarded as a biomarker of violent behavior in male adolescents. The high classifyication accuracy demonstrates that abnormal brain structure may be a partial cause of violent behaviors.

\section{Limitations}


314 There are several limitations in this study. (1) This study was conducted solely in male 315 adolescents. Thus, our findings cannot be generalized to females, adults, and children younger 316 than 14 years old. (2) In our study setting, we only have two classes: adolescents with violent

317 behaviors versus adolescents without violent behaviors. That is to say, different types of violent

318 behaviors are treated as the same class, i.e., violent behavior. As a result, only classification 319 experiment analysis was conducted. Nevertheless, it is feasible to quantify different types of 320 violent behaviors by assigning different scores according to the severity. Further correlation 321 analysis based on the scores may help uncover more findings. (3) The study is based on a small 322 cohort of 59 participants. Additional experiments on other independent samples/cohort can help 323 further validate our findings. However, as mentioned in our introduction, little work has been 324 done to investigate the association of abnormal brain with violence tendency in healthy male 325 adolescents. Therefore, at the moment we are not able to find an independent cohort to validate 326 the findings. (4) Several other factors, e.g., stress and personality traits, are also related to 327 violence tendency. However, the information of these personality traits were not available in the 328 current cohort, which limited the conclusion of our work.

329

330

\section{Conclusions}

331 We found a reduced GMV in 5 different brain regions in male adolescent offenders compared to 332 that in male adolescent HCS. Moreover, our analyses verified the validity and practicality of using structural neuroimaging analyses to distinguish violent adolescent offenders and nonviolent adolescents in males. Specifically, VBM technique is helpful for characterizing violent male adolescents. In addition, the findings in this study suggest that reduced volume in the frontal-temporal-parietal-subcortical circuit may be closely associated with violent behaviors in male adolescents, and thus could represent an important potential biomarker for detecting violent behavioral tendencies in male adolescents.

\section{Acknowledgements}

341 Not applicable.

342

\section{Funding:}


344 This study was supported by National Natural Science Foundation of China (NO. 30800368, 345 81371500, 81571341 and 81501637), and the MOE (Ministry of Education in China) Project of 346 Humanities and Social Sciences (Project No. 13YJC190033).

347

348

349

350

351

352

353

354

355

356

357

358

359

360

361

362

363

364

365

366

367

368

369

370

371

372

373

374

375

\section{Authors' contributions:}

XPW worked on study conception and design; YDZ collected, analyzed, interpreted the data and drafted the manuscript; JSZ, FML and XPW critically revised the manuscript; All authors have read and approved the final draft.

\section{Human Ethics:}

All study procedures were approved by Institutional Review Board of the second Xiangya Hospital, Central South University.

\section{Conflicts of Interests:}

Authors declare no conflicts of interests.

\section{References}

Barkataki I, Kumari V, Das M, et al. 2006. Volumetric structural brain abnormalities in men with schizophrenia or antisocial personality disorder. Behavioural brain research, 169: 239-247. Bertsch K, Grothe M, Prehn K, et al. 2013. Brain volumes differ between diagnostic groups of violent criminal offenders. European archives of psychiatry and clinical neuroscience, 263: 593606.

Boes AD, Bechara A, Tranel D, et al. 2009. Right ventromedial prefrontal cortex: a neuroanatomical correlate of impulse control in boys. Social cognitive and affective neuroscience, 4: 1-9.

Boes AD, Tranel D, Anderson SW, et al. 2008. Right anterior cingulate: a neuroanatomical correlate of aggression and defiance in boys. Behavioral neuroscience, 122: 677-684.

Bussing R, Grudnik J, Mason D, et al. 2002. ADHD and conduct disorder: an MRI study in a community sample. The world journal of biological psychiatry : the official journal of the World Federation of Societies of Biological Psychiatry, 3: 216-220.

De Brito SA, Mechelli A, Wilke M, et al. 2009. Size matters: increased grey matter in boys with conduct problems and callous-unemotional traits. Brain : a journal of neurology, 132: 843-852. 
376 de Oliveira-Souza R, Hare RD, Bramati IE, et al. 2008. Psychopathy as a disorder of the moral

377 brain: fronto-temporo-limbic grey matter reductions demonstrated by voxel-based morphometry. 378 NeuroImage, 40: 1202-1213.

379 Ducharme S, Hudziak JJ, Botteron KN, et al. 2011. Right anterior cingulate cortical thickness 380 and bilateral striatal volume correlate with child behavior checklist aggressive behavior scores in 381 healthy children. Biological psychiatry, 70: 283-290.

382 Ermer E, Cope LM, Nyalakanti PK, et al. 2012. Aberrant paralimbic gray matter in criminal 383 psychopathy. Journal of abnormal psychology, 121: 649-658.

384 Ermer E, Cope LM, Nyalakanti PK, et al. 2013. Aberrant paralimbic gray matter in incarcerated 385 male adolescents with psychopathic traits. Journal of the American Academy of Child and 386 Adolescent Psychiatry, 52: 94-103.e103.

387 Fairchild G, Hagan CC, Walsh ND, et al. 2013. Brain structure abnormalities in adolescent girls 388 with conduct disorder. Journal of child psychology and psychiatry, and allied disciplines, 54: 8638995.

390 Fairchild G, Passamonti L, Hurford G, et al. 2011. Brain structure abnormalities in early-onset 391 and adolescent-onset conduct disorder. The American journal of psychiatry, 168: 624-633.

392 Gansler DA, McLaughlin NC, Iguchi L, et al. 2009. A multivariate approach to aggression and 393 the orbital frontal cortex in psychiatric patients. Psychiatry research, 171:145-154.

394 Giedd JN, Denker AH. 2015. The Adolescent Brain: Insights from Neuroimaging. Springer 395 International Publishing.

396 Grafman J, Schwab K, Warden D, et al. 1996. Frontal lobe injuries, violence, and aggression: a 397 report of the Vietnam Head Injury Study. Neurology, 46: 1231-1238.

398 Greenwood P. 2008. Prevention and intervention programs for juvenile offenders. The Future of 399 children, 18: 185-210.

400 Gregory S, ffytche D, Simmons A, et al. 2012. The antisocial brain: psychopathy matters.

401 Archives of general psychiatry, 69: 962-972.

402 Huebner T, Vloet TD, Marx I, et al. 2008. Morphometric brain abnormalities in boys with 403 conduct disorder. Journal of the American Academy of Child and Adolescent Psychiatry, 47: $404540-547$.

405 Husain FT, Medina RE, Davis CW, et al. 2011. Neuroanatomical changes due to hearing loss 406 and chronic tinnitus: a combined VBM and DTI study. Brain research, 1369: 74-88. 
407 Jednorog K, Gawron N, Marchewka A, et al. 2014. Cognitive subtypes of dyslexia are

408 characterized by distinct patterns of grey matter volume. Brain structure \& function, 219: 1697 4091707.

410 Kruesi MJ, Casanova MF, Mannheim G, et al. 2004. Reduced temporal lobe volume in early 411 onset conduct disorder. Psychiatry research, 132: 1-11.

412 Krug EG, Mercy JA, Dahlberg LL, et al. 2002. The world report on violence and health. The 413 Lancet, 360: 1083-1088.

414 Kumari V, Barkataki I, Goswami S, et al. 2009. Dysfunctional, but not functional, impulsivity is 415 associated with a history of seriously violent behaviour and reduced orbitofrontal and 416 hippocampal volumes in schizophrenia. Psychiatry research, 173: 39-44.

417 Laakso MP, Gunning-Dixon F, Vaurio O, et al. 2002. Prefrontal volumes in habitually violent 418 subjects with antisocial personality disorder and type 2 alcoholism. Psychiatry research, 114:95419102.

420 Lane SD, Kjome KL, Moeller FG. 2011. Neuropsychiatry of aggression. Neurologic clinics, 29: 421 49-64, vii.

422 Leutgeb V, Wabnegger A, Leitner M, et al. 2016. Altered cerebellar-amygdala connectivity in 423 violent offenders: A resting-state fMRI study. Neuroscience letters, 610: 160-164.

424 Linden A. 2006. Measuring diagnostic and predictive accuracy in disease management: an 425 introduction to receiver operating characteristic (ROC) analysis. Journal of evaluation in clinical 426 practice, 12: 132-139.

427 Matsuo K, Nicoletti M, Nemoto K, et al. 2009a. A voxel-based morphometry study of frontal 428 gray matter correlates of impulsivity. Human brain mapping, 30: 1188-1195.

429 Matsuo K, Nicoletti MA, Peluso MA, et al. 2009b. Anterior cingulate volumes associated with 430 trait impulsivity in individuals with bipolar disorder. Bipolar disorders, 11: 628-636.

431 Matthies S, Rusch N, Weber M, et al. 2012. Small amygdala-high aggression? The role of the 432 amygdala in modulating aggression in healthy subjects. The world journal of biological 433 psychiatry : the official journal of the World Federation of Societies of Biological Psychiatry, 13: $434 \quad 75-81$.

435 McKinlay A, Corrigan J, Horwood LJ, et al. 2014. Substance abuse and criminal activities 436 following traumatic brain injury in childhood, adolescence, and early adulthood. J Head Trauma 437 Rehabil. 29:498-506. 
438 Muller JL, Ganssbauer S, Sommer M, et al. 2008. Gray matter changes in right superior temporal 439 gyrus in criminal psychopaths. Evidence from voxel-based morphometry. Psychiatry research, 440 163: 213-222.

441 Pardini DA, Raine A, Erickson K, et al. 2014. Lower amygdala volume in men is associated with 442 childhood aggression, early psychopathic traits, and future violence. Biological psychiatry, 443 75:73-80.

444 Perathoner S, Cordero-Maldonado ML, Crawford AD, 2016. Potential of zebrafish as a model 445 for exploring the role of the amygdala in emotional memory and motivational behavior. Journal 446 of neuroscience research, 94: 445-462.

447 Puri BK, Counsell SJ, Saeed N, et al. 2008. Regional grey matter volumetric changes in forensic 448 schizophrenia patients: an MRI study comparing the brain structure of patients who have 449 seriously and violently offended with that of patients who have not. BMC psychiatry, 8:S6.

450 Raine A, Lencz T, Bihrle S, et al. 2000. Reduced prefrontal gray matter volume and reduced 451 autonomic activity in antisocial personality disorder. Archives of general psychiatry, 57: 119-127; 452 discussion 128-119.

453 Rosell DR, Siever LJ, 2015. The neurobiology of aggression and violence. CNS spectrums 20(3), 454 254-279.

455 Santillo AF, Martensson J, Lindberg O, et al. 2013. Diffusion tensor tractography versus 456 volumetric imaging in the diagnosis of behavioral variant frontotemporal dementia. PloS one, 8: 457 e66932.

458 Schiffer B, Muller BW, Scherbaum N, et al. 2011. Disentangling structural brain alterations 459 associated with violent behavior from those associated with substance use disorders. Archives of 460 general psychiatry, 68: 1039-1049.

461 Shanee N, Apter A, Weizman A, 1997. Psychometric properties of the K-SADS-PL in an Israeli 462 adolescent clinical population. The Israel journal of psychiatry and related sciences, 34: 179-186. 463 Soderstrom H, Hultin L, Tullberg M, et al. 2002. Reduced frontotemporal perfusion in 464 psychopathic personality. Psychiatry research, 114:81-94.

465 Sowell ER, Thompson PM, Tessner KD, et al. 2001. Mapping continued brain growth and gray 466 matter density reduction in dorsal frontal cortex: Inverse relationships during postadolescent 467 brain maturation. Journal of Neuroscience, 21: 8819-8829.

468 Sterzer P, Stadler C, 2009. Neuroimaging of aggressive and violent behaviour in children and 
469 adolescents. Frontiers in behavioral neuroscience, 3: 35.

470 Sterzer P, Stadler C, Poustka F, et al. 2007. A structural neural deficit in adolescents with

471 conduct disorder and its association with lack of empathy. NeuroImage, 37: 335-342.

472 Stevens MC, Haney-Caron E, 2012. Comparison of brain volume abnormalities between ADHD

473 and conduct disorder in adolescence. Journal of psychiatry \& neuroscience : JPN, 37: 110148.

474 Tiihonen J, Rossi R, Laakso MP, et al. 2008. Brain anatomy of persistent violent offenders: more

475 rather than less. Psychiatry research, 163: 201-212.

476 Viding E, Blair RJ, Moffitt TE, et al. 2005. Evidence for substantial genetic risk for psychopathy

477 in 7-year-olds. Journal of child psychology and psychiatry, and allied disciplines, 46: 592-597.

478 Vloet TD, Konrad K, Huebner T, et al. 2008. Structural and functional MRI- findings in children

479 and adolescents with antisocial behavior. Behavioral sciences \& the law, 26: 99-111.

480 Yang Y, Raine A, Han CB, et al. 2010. Reduced hippocampal and parahippocampal volumes in

481 murderers with schizophrenia. Psychiatry research, 182: 9-13.

482 Yang Y, Raine A, Lencz T, et al. 2005. Volume reduction in prefrontal gray matter in

483 unsuccessful criminal psychopaths. Biological psychiatry, 57:1103-1108.

484 Zhang L, Kerich M, Schwandt ML, et al. 2013. Smaller right amygdala in Caucasian alcohol-

485 dependent male patients with a history of intimate partner violence: a volumetric imaging study.

486 Addiction biology, 18: 537-547. 
Figure 1

Segmentation results of one typical subject.

These results are obtained in the original space of the images. (A) segmented grey matter (c1X.img); (B) segmented white matter (c2X.img); (C) segmented CSF (c3X.img); (D) segmented skull (c4X.img). CSF= Cerebrospinal Fluid. 
A. Grey matter
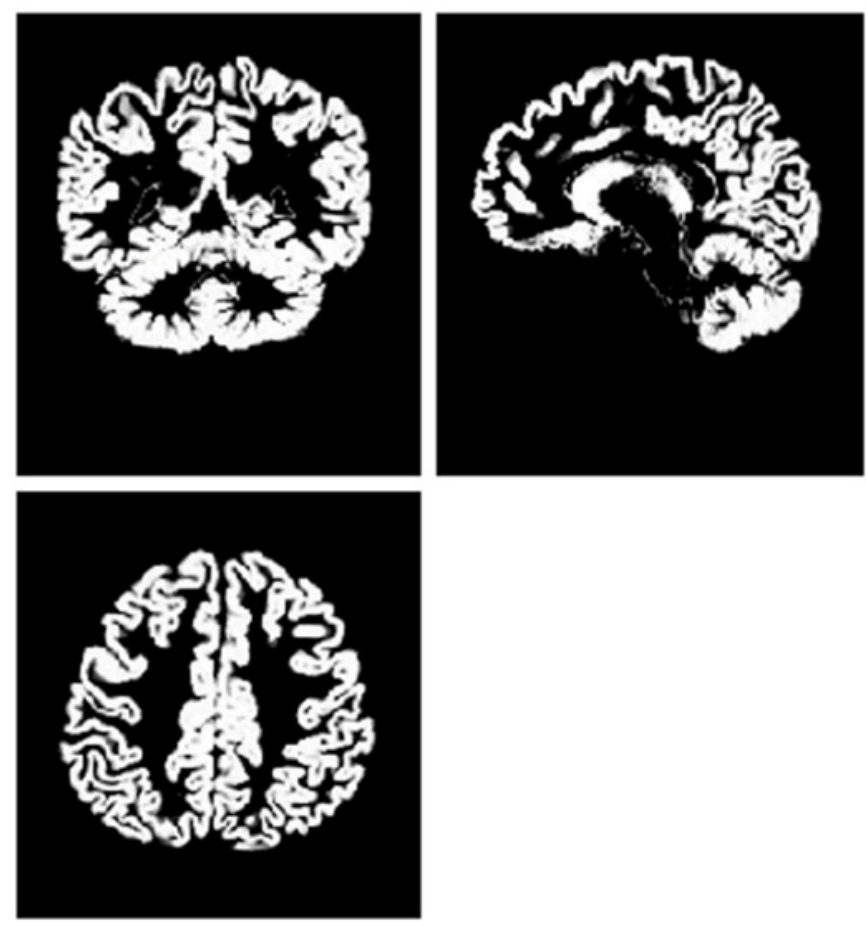

\section{CSF}
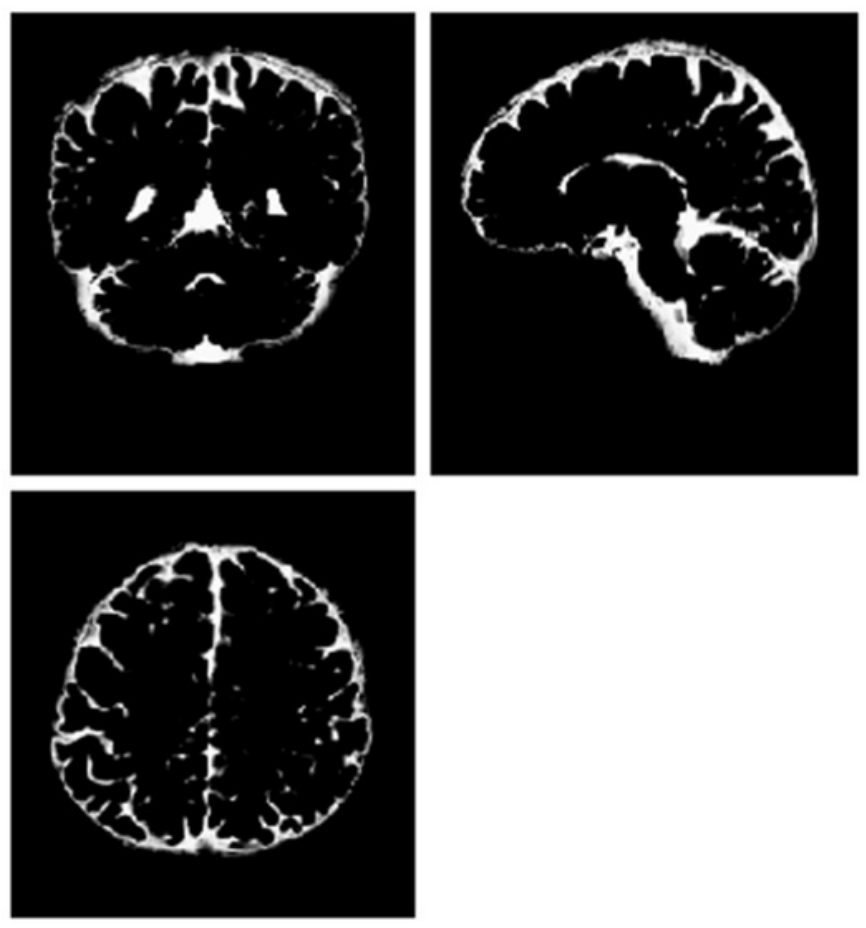

B. White matter
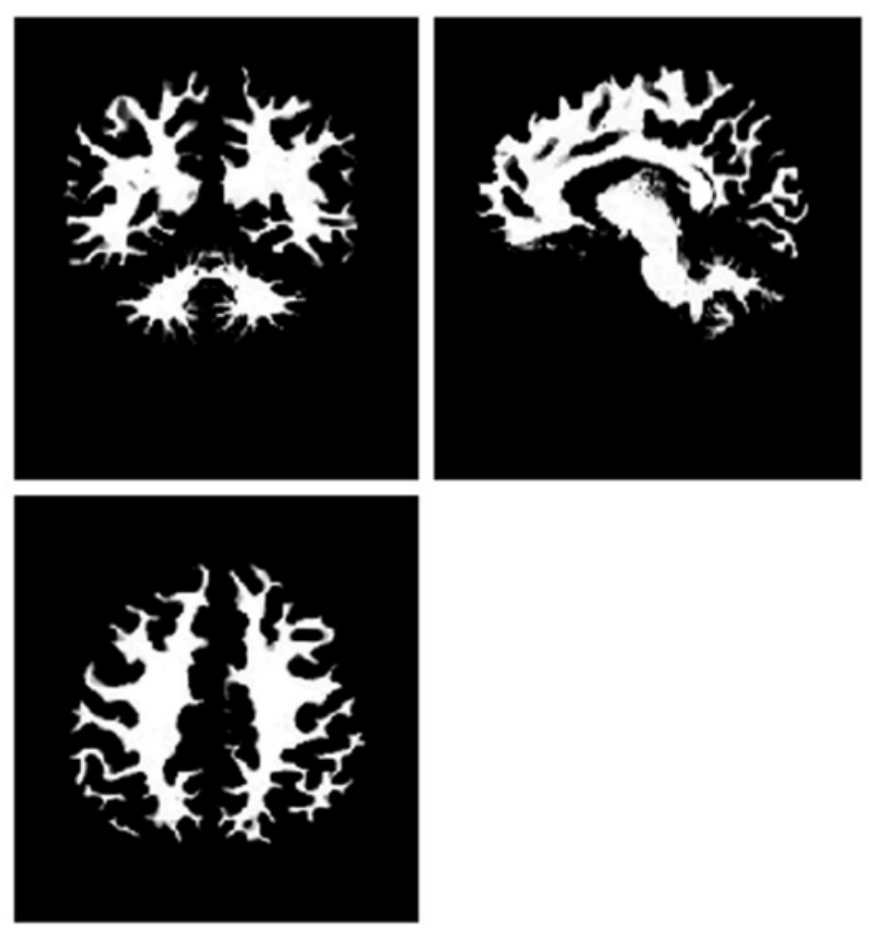

D. Skull
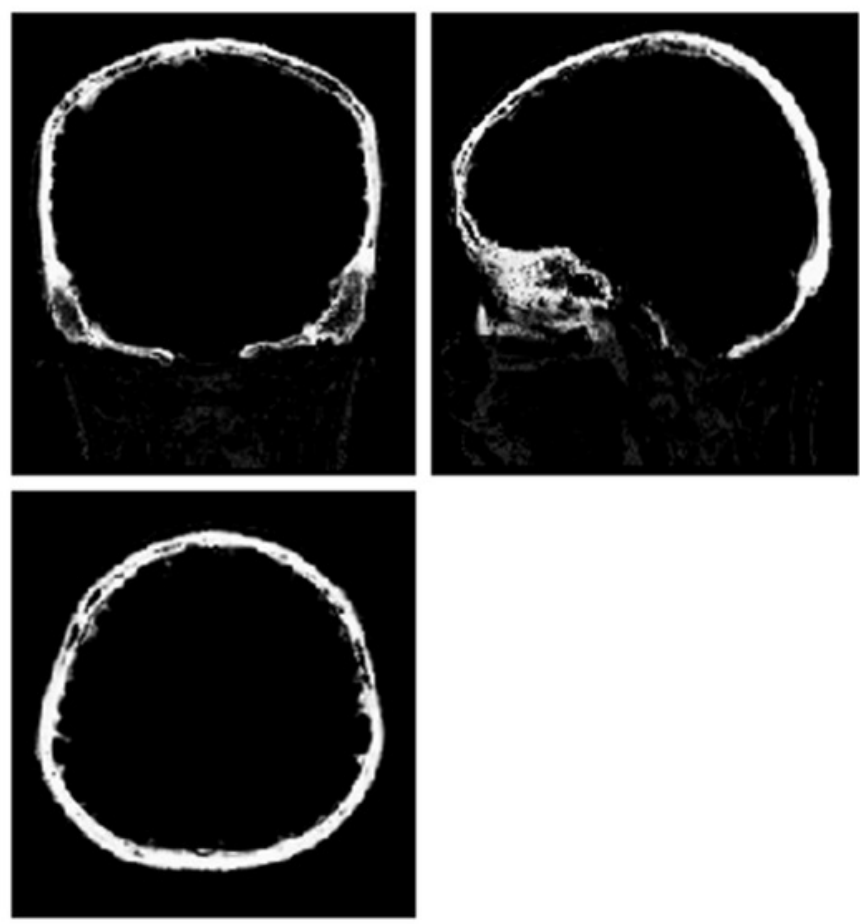


\section{Figure 2}

Brain voxel-based morphometry showing lower gray matter volume in male violent adolescent offenders superimposed on a T1-weightedtemplate (violent adolescent offenders vs. controls; two-sample t-tests).

These brain areas include the left olfactory cortex $(A, B)$, the left amygdala $(C, D)$, the left middle temporal gyrus $(C, D)$, the left inferior parietal lobe $(E)$, and the right superior temporal gyrus $(F)$. The color bar indicates the $t$ value of the between-group analysis.
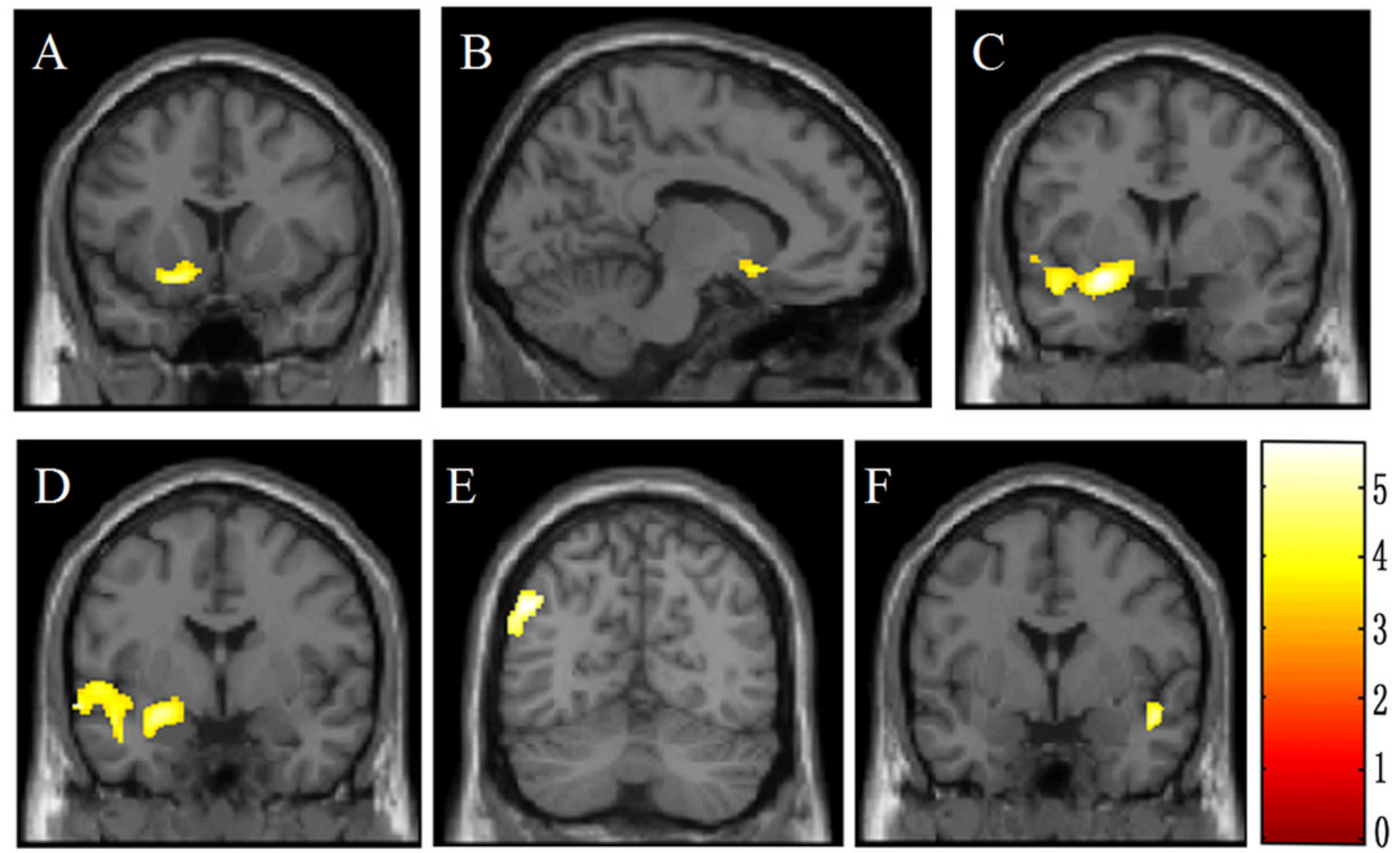


\section{Table 1 (on next page)}

Sociodemographic and clinical characteristics (mean \pm S.D.) in adolescent offenders and healthy controls (two-sample $t$-tests). 
1

\begin{tabular}{|c|c|c|c|c|}
\hline & HC & AO & Statistic & P-value \\
\hline Age, years & $\mathbf{N = 2 9 )}$ & $17.7 \pm 0.8$ & $t=0.45$ & 0.66 \\
\hline Literacy, years & $10.0 \pm 0.0$ & $7.5 \pm 2.4$ & $t=-5.10$ & 0.00 \\
\hline TIV, $\mathrm{mm}^{3}$ & $1335 \pm 86$ & $1348 \pm 107$ & $t=0.45$ & 0.65 \\
\hline GMV, $\mathrm{mm}^{3}$ & $633 \pm 39$ & $629 \pm 46$ & $t=0.84$ & 0.72 \\
\hline WMV, $\mathrm{mm}^{3}$ & $462 \pm 38$ & $473 \pm 50$ & 0.40 \\
\hline
\end{tabular}

2 Abbreviations: S.D.=standard deviation; $\mathrm{HC}=$ healthy controls; $\mathrm{AO}=$ adolescent offenders; $\mathrm{TIV}=\mathrm{Total}$

3 intracranial volume; GMV=gray matter volume; WMV=white matter volume.

4 


\section{Table 2 (on next page)}

Regions with smaller gray matter volume in adolescent offenders $(\mathrm{N}=31)$ compared with healthy controls $(\mathrm{N}=29)$, measured using voxel-based morphometry (corrected false discovery rate at $P \square 0.05) *$. 
1

\begin{tabular}{|c|c|c|c|c|c|c|c|}
\hline \multirow[t]{2}{*}{ Regions } & \multirow[t]{2}{*}{$\mathbf{B A}$} & \multicolumn{3}{|c|}{$\begin{array}{l}\text { Stereotactic coordinates } \\
(\mathrm{mm})\end{array}$} & \multirow{2}{*}{$\begin{array}{c}\text { Cluster } \\
\text { size } \\
\left(\mathrm{mm}^{3}\right)\end{array}$} & \multirow[t]{2}{*}{$\mathbf{T}$} & \multirow[t]{2}{*}{$P$-value } \\
\hline & & $\mathrm{X}$ & $\mathrm{y}$ & $\mathrm{Z}$ & & & \\
\hline $\mathrm{L} \mathrm{OC}$ & 11 & -17 & 12 & -14 & 232 & -4.68 & $0.000 * *$ \\
\hline $\mathrm{L} A \mathrm{AMG}$ & 34 & -26 & 3 & -15 & 191 & -6.50 & 0.014 \\
\hline L MTG & 21 & -65 & -1 & -11 & 103 & -5.12 & 0.047 \\
\hline L IPL & 39 & -39 & -52 & 54 & 130 & -4.43 & $0.000 * *$ \\
\hline R STG & 21 & 40 & 0 & -15 & 104 & -4.72 & $0.000 * *$ \\
\hline
\end{tabular}

2 Abbreviations: $\mathrm{BA}=$ Brodmann's area; $\mathrm{T}=$ intensity; $\mathrm{L}=\mathrm{left}$; $\mathrm{R}=\mathrm{right}$; $\mathrm{OC}=\mathrm{olfactory}$ cortex;

$3 \mathrm{AMG}=$ amygdala; $\mathrm{MTG}=$ middle temporal gyrus; $\mathrm{IPL}=$ inferior parietal lobe; $\mathrm{STG}=$ superior temporal gyrus

$4 *$ The duration of education was select as the covariant.

$5 * *$ Uncorrected false discovery rate.

6 


\section{Table 3(on next page)}

Area under the curve (AUC) details for the five brain regions of interest showing significant differences in gray matter volume between male adolescent offenders and male age-matched controls. 
1

\begin{tabular}{|c|c|c|c|c|c|}
\hline & L OC & L AMG & L MTG & L IPL & R STG \\
\hline Sensitivity\% & 58.6 & 86.2 & 79.3 & 86.2 & 89.7 \\
\hline Specificity\% & 67.7 & 74.2 & 58.1 & 74.2 & 64.5 \\
\hline AUC & 0.642 & 0.834 & 0.721 & 0.834 & 0.790 \\
\hline
\end{tabular}

2 Abbreviations: $\mathrm{L}=\mathrm{left}$; $\mathrm{R}=$ right; $\mathrm{OC}=$ olfactory cortex; $\mathrm{AMG}=$ amygdala; $\mathrm{MTG}=$ middle temporal gyrus;

$3 \mathrm{IPL}=$ inferior parietal lobe; $\mathrm{STG}=$ superior temporal gyrus; $\mathrm{AUC}=\mathrm{Area}$ under the curve

4 